\title{
GESTIÓN DE LIBROS GENEALÓGICOS EN LAS RAZAS DE GALLINAS CATALANAS
}

\author{
HERD BOOK MANAGEMENT IN CATALAN CHICKEN BREEDS
}

\author{
Cartañà, M. ${ }^{1}$ y Francesch, A. ${ }^{1 *}$
}

IIRTA. Centre Mas de Bover. Constantí. Tarragona. España. *Amadeu.Francesch@irta.cat

\section{Palabras clave adicionales}

Control genealógico. Inseminación artificial. Pedigrí. Razas de gallinas autóctonas.

\section{RESUMEN}

En el 2008 se crearon los Libros Genealógicos de las razas de gallinas catalanas cuya gestión lleva el IRTA. Para ello se utilizaron dos sistemas algo distintos según la Asociación de Criadores, los que damos a conocer dado que ambos permiten un control muy estricto del pedigrí, que como es sabido resulta difícil en aves.

Los adultos inscritos en los Registros Fundacional, Definitivo o Auxiliar, en el momento de la reproducción, se individualizaron en baterías. EI IRTA diseñó los apareamientos y reprodujo los individuos seleccionados mediante inseminación artificial.

En el caso de la Asociación de la raza Prat, los criadores están centralizados en la zona de origen y los reproductores están en sus explotaciones desde el día de vida. En el momento de la reproducción se recogieron los huevos numerados, se transportaron al IRTA donde tuvo lugar el nacimiento, la identificación y la inscripción en el Registro de Nacimientos de los nuevos ejemplares. La cría posterior y la selección se realizaron en las explotaciones de los socios. En el momento actual figuran inscritos 175 machos y 461 hembras.

En las Asociaciones de las razas Penedesenca y Empordanesa los socios que participan están muy dispersos. En este caso la cría, selección y reproducción se realizó en el IRTA. Una vez reproducidos los selectos, se suministraron a los criadores, los cuales continuaron con la conservación. Actualmente, en el libro de la raza

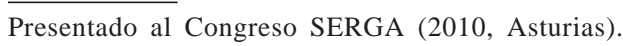

\section{AdDITIONAL KEYWORDS}

Genealogical control. Artificial insemination. Pedigree. Local chicken breeds.

Penedesenca figuran inscritos 204 machos y 420 hembras, mientras que en el de la Empordanesa hay 229 machos y 527 hembras.

\section{SUMMARY}

In 2008 the herd books of Catalan chicken breeds were created and their management is conducted by IRTA. We present two different management systems which are being used depending on breeders associations; both allow us a strict control of the pedigree, it is well known that is difficult in birds.

The adults registered in the Foundational, Definitive or Auxiliary Registers, at the moment of the reproduction, they were housed on individual cages. IRTA designed the matings and reproduced the selected birds by artificial insemination.

In Prat breed Association, the partners are situated in the origin zone and the breeders are in the farms since the birth day. At the moment of the reproduction, the partners collected the numbered eggs and they were transported to IRTA where the birth, the identification and the register of the newborns in the Birth Register took place. The rearing and the selection were carrying out in the breeder's farms. In that moment there are 175 males and 461 females registered.

In Penedesenca and Empordanesa breed Associations, the partners are widely dispersed. In this case rearing, selection and reproduction were carrying out in IRTA. When selected birds were reproduced, they were provided to the breeders who continued with the conservation. 


\section{CARTAÑÀYFRANCESCH}

At present, in Penedesenca breed herd book there are 204 males and 420 females and in Empordanesa breed herd book there are 229 males and 527 females.

\section{INTRODUCCIÓN}

En 1985, en el IRTA, se empezaron los programas de recuperación de las razas de gallinas Prat, Penedesenca y Empordanesa (Francesch, 1998). Estas razas están consideradas en peligro de extinción dentro del Catálogo Oficial de Razas de Ganado de España contenido en el Real Decreto 2129/ 2008 , de 26 de diciembre, lo que hizo posible la creación de los libros genealógicos por el DAR y permitió establecer, para su gestión, una relación más estrecha del IRTA con las Asociaciones de Criadores con el fin de caracterizarlas y mejorarlas. Al mismo tiempo ha facilitado la descentralización de su conservación y extender la población del IRTA a las explotaciones de criadores, aumentando así, la seguridad de su conservación.

Esta actividad permite un control estricto de los pedigrís, además de disponer de los datos necesarios para poder desarrollar programas de mejora.

La relación IRTA-Asociación es distinta según las características de cada asociación. Estas diferencias han puesto en evidencia la necesidad de llevar a cabo una gestión distinta dependiendo de la asociación, las cuales damos a conocer ya que permiten un control muy estricto del pedigrí, que sabemos que no es fácil en aves, como podemos comprobar en otros trabajos en los que se describen libros genealógicos en gallinas (Rois, 2008; Mercadal y Marqués, 2008).

Por tanto, el objetivo del presente trabajo es presentar como se han constituido los libros genealógicos de las razas de gallinas catalanas y ver los esquemas de funcionamiento iniciados, que esperamos puedan continuar en las sucesivas generaciones, años tras año.

\section{MATERIAL Y MÉTODOS}

\section{CONSTITUCIÓNDEL REGISTRO FUNDACIONAL}

Para la creación de los libros se partió del stock de animales presentes en el IRTA, que como es conocido (Francesch, 2010) estaban formados por variedades o subpoblaciones cerradas desde 1986, cuatro en la raza Penedesenca, cinco en la Empordanesa y dos en la Prat. Los gallos y gallinas de estas poblaciones fueron valorados morfológicamente teniendo en cuenta el patrón y la calificación morfológica de acuerdo con la normativa, gallos con 70 puntos o más y gallinas con 65 puntos o más pasaron a formar parte del Registro Fundacional (RF).

\section{CONSTITUCIÓN DEL REGISTRO DE NACI- MIENTOS}

Una vez creado el RF se procedió a la reproducción. Para ello, tanto gallos como gallinas se ubicaron en baterías individualizadas y numeradas. Se diseñaron apareamientos de un gallo por cada tres o cuatro gallinas teniendo en cuenta el pedigrí de cada uno con el fin de evitar al máximo la consanguinidad. Los apareamientos se realizaron mediante inseminación artificial en las instalaciones del IRTA. Los huevos recogidos se marcaron con el número de la batería de la gallina. Para la incubación, los huevos con el mismo número fueron agrupados para facilitar así el nacimiento de las familias completas en el mismo compartimento. En el nacimiento los pollitos fueron identificados mediante una banda numerada insertada en la membrana alar. Con éste número se inscribieron en el Registro de Nacimientos (RN) asociados a un padre y a una madre.

En las Asociaciones de la Penedesenca y de la Empordanesa los criadores que participan en la gestión del Libro Genealógico estaban muy dispersos, y esto dificultó el trabajo coordinado entre explotaciones. Por esto se desarrolló una relación IRTA-asociación en la cual los pollitos recién naci- 
dos, identificados e inscritos en el Registro de Nacimientos (RN) se criaran en el IRTA.

En cambio, en el caso de la Asociación de la Prat los criadores estaban centralizados en la zona de origen y esto permitió que los pollitos entraran en las explotaciones cuando sólo tenían un día de vida. No obstante, en el IRTA se continuó la cría y selección de una población que también ha quedado inscrita y que se utiliza como madre para la obtención del pollo Prat mejorado.

\section{CONSTITUCIÓN DE LOS REGISTROS DEFI- NITIVOY AUXILIAR}

La valoración morfológica de los gallos y las gallinas se realizó a las 20 semanas de vida por técnicos del IRTA y miembros de las Asociaciones de Criadores. En este momento se creó el Registro Definitivo (RD) donde se inscribieron los individuos seleccionados que tenían el pedigrí totalmente conocido y también se creó el Registro Auxiliar (RA) donde se inscribieron los que perdieron total o parcialmente el pedigrí.

\section{CONTROL DE PARÁMETROS PRODUCTIVOSY EVALUACIONESGENÉTICAS}

Los inscritos en los RD y RA de las poblaciones mantenidas en el IRTA se sometieron, como ya era habitual, a control de los parámetros productivos siguientes: peso del animal vivo a las 11 semanas de vida, peso del huevo y color de la cáscara a las 27 semanas y número de huevos por gallina hasta las 39 semanas de vida.

Terminados los controles y como ya también era habitual (Francesch, 2002) los animales fueron evaluados genéticamente mediante un modelo animal multicaracter.

Esto permitió, como de costumbre y dentro de lo posible, hacer una mínima selección en las gallinas y una selección algo más estricta en los gallos en base a los valores BLUP dando preferencia a aquellos caracteres que les son propios, como el color intenso de la cáscara del huevo en las razas Penedesenca y Empordanesa.

\section{ProseguimientodelaActividad}

Terminados todos los controles morfológicos y productivos se decidieron los gallos y gallinas que iban a ser los reproductores. Una vez seleccionados, se prepararon los apareamientos con un gallo por cada tres gallinas impidiendo los apareamientos hasta el nivel de medio hermano. A partir de estos se hicieron las inseminaciones con la colaboración de los criadores.

En el caso de la Asociación de la Prat fueron los mismos criadores los que se encargaron de la recogida de los huevos y los marcaron con el número de batería de la gallina. Estos huevos se transportaron al IRTA, donde se incubaron junto con los huevos de las razas Penedesenca, Empordanesa y la Prat en mejora recogidos en el IRTA, donde tuvo lugar el nacimiento. Los pollitos fueron anillados e inscritos en el RN.

A continuación parte de los pollitos Prat fueron entregados a los criadores de la Asociación del Prat y los que formaban parte de la población de mejora quedaron en el IRTA.

En el caso de las asociaciones de las razas Penedesenca y Empordanesa los pollitos se quedaron en las instalaciones del IRTA. Mientras que por otra parte, los criadores se hicieron cargo de los progenitores que ubicaron en sus explotaciones donde continuó la conservación y difusión de las razas mediante nuevas incubaciones realizadas por los criadores aunque al margen del registro genealógico. Como ya era habitual esta labor de difusión, ahora con los ejemplares inscritos en los RD y RA, también la realizó la asociación de la Prat mediante incubaciones controladas por ellos.

INFORMACIÓN CONTENIDAENLOSREGISTROS

Todos los registros contienen la siguiente información: $n^{\circ}$ de anilla del animal, $n^{\circ}$ de anilla del padre, $\mathrm{n}^{\circ}$ de anilla de la madre, variedad, sexo, batería, fecha de nacimiento, fecha de baja, causa de la baja, observa- 


\section{CARTAÑÀYFRANCESCH}

ciones, puntación morfológica, criador, valores fenotípicos de puesta, peso del huevo, color de la cáscara del huevo y peso vivo, además de los valores genéticos BLUP de dichos parámetros obtenidos en la evaluación realizada después de los controles.

\section{RESULTADOS}

En la raza Prat se inscribieron al RF un total de 57 machos y 212 hembras, que dieron lugar a 887 pollitos que se inscribieron en el RN de los cuales 253 pasaron al RD y 111 al RA. Actualmente en los RN, RD y RA tenemos un total de 175 machos y 461 hembras.

En la raza Penedesenca se inscribieron al RF un total de 69 machos y 284 hembras, de estos nacieron 797 pollitos, de los cuales 299 pasaron al RD y 1 al RA. Actualmente en

\section{BIBLIOGRAFÍA}

Francesch, A. 1998. Funcionamiento de la conservación de razas de gallinas autóctonas en Cataluña. Primer Congreso Nacional de SERGA 14-17 diciembre 1997. Córdoba. España. Arch. Zootec., 47: 141-148.

Francesch, A. 2002. Mejora genética de razas de gallinas catalanas. ITEA, (98A): 173-184.

Francesch, A. 2010. Races avícoles. Dossier los RN, RD y RA tenemos un total de 204 machos y 420 hembras.

En la raza Empordanesa se inscribieron al RF un total de 86 machos y 260 , la reproducción de los cuales permitió obtener 963 pollitos que se inscribieron en el RN de los cuales 353 pasaron al RD y 7 al RA. Actualmente en los RN, RD y RA tenemos un total de 229 machos y 527 hembras.

\section{AGRADECIMIENTOS}

La labor presentada ha sido posible gracias a las ayudas recibidas del DAR de la Generalitat de Cataluña y del INIA dentro del proyecto RZP2008-00003-00-00, así como a la colaboración de la Asociación de Criadores Prat, de la Asociación de Criadores Penedesenca y de la Asociación de Criadores Empordanesa.

Tècnic., 44: 13-23.

Mercadal, J. y Marqués, N. 2008. Control del censo en la Gallina Menorca mediante Registro Genealógico. Il Congreso de Etnología Avícola. 16-18 Abril 2008. Barcelona. España.

Rois, D. 2008. Funcionamiento del Libro de Registro de la Galina de Mos. II Congreso de Etnología Avícola. 16-18 Abril 2008. Barcelona. España. 